\title{
A Escada Educacional do Design Thinking no Brasil: revisão sistemática nos Anais do P\&D
}

\section{The Design Thinking's Educational Ladder in Brazil: systematic review at P\&D's proceedings}

\author{
Marina Arakaki, Unesp - Universidade Estadual Paulista \\ marina.arakaki@unesp.br
}

\author{
Ana Cláudia de Abreu, Unesp - Universidade Estadual Paulista \\ a.abreu@unesp.br
}

Mariano Lopes de Andrade Neto, UniFSP - Centro Universitário Sudoeste Paulista mlaneto@gmail.com

\begin{abstract}
Resumo
O termo design thinking tem sido discutido e estudado desde 1992 como uma metodologia para resolução de problemas, passando por diferentes formas de abordagem e procedimentos ao longo dos anos. Esses processos foram sistematizados na escada dinamarquesa do design, desenvolvida pelo Danish Design Centre, em 2001. Inspirado nesse modelo, foi desenvolvido em 2017, a Escada Educacional do Design, que ilustra os estágios pedagógicos no desenvolvimento do design thinking. Dessa forma, o objetivo deste estudo é investigar os níveis de aplicação do design thinking, de acordo com a Escada Educacional do Design, nos Anais do Congresso Brasileiro de Pesquisa e Desenvolvimento em Design (P\&D), buscando configurar sua representatividade no mesmo. Como resultado, obteve-se a visualização dos níveis trabalhados da Escada Educacional do Design nesses anais, além de um panorama da disciplina no congresso e a identificação de lacunas para novas pesquisas serem realizadas.
\end{abstract}

Palavras-chave: design thinking, escada educacional do design, P\&D Brasil

\begin{abstract}
The expression design thinking has been discussed and studied since 1992, as a methodology for problem-solving, going through different approaches and procedures over the years. These processes were systematized at the Danish design ladder, developed by the Danish Design Centre, in 2001. Inspired by this model, the Educational Design Ladder was developed in 2017, that illustrates the pedagogical stages in the design thinking development. Thus, the aim of this study is to investigate the design thinking application levels, according to the Educational Design Ladder, in the Congresso Brasileiro de Pesquisa e Desenvolvimento em Design (P\&D)'s proceedings, seeking to configure its representativeness in it. As a result, it was obtained the visualization of the Educational Design Ladder worked levels in these proceeding, besides na overview of the discipline in the congress and the identification of gaps for new researches to be carried out.
\end{abstract}

Keywords: design thinking, educational design ladder, $P \& D$ Brazil 
estudos EM DEsign

1 DESIGN ARTICLES 


\section{Introdução}

Cada vez mais utilizado no mundo dos negócios e projetos, o termo design thinking desperta dúvidas e curiosidade. Surgido em 1992 no meio acadêmico, tornou-se rapidamente uma abordagem alternativa para designers e não-designers.

No Brasil, o primeiro livro sobre o tema foi publicado em 2010, escrito por Tim Brown, com o título Design Thinking: uma metodologia poderosa para decretar o fim das velhas ideias. No mesmo ano foi lançado o livro Design de Negócios: por que o design thinking se tornará a próxima vantagem competitiva dos negócios e como se beneficiar disso, de Roger Martin. O primeiro livro brasileiro foi lançado em 2011, Design Thinking Brasil, de Tennyson Pinheiro e Luis Alt.

Quanto ao ensino, o primeiro curso de Inovação \& Design Thinking da América Latina foi criado em 2010, na Escola Superior de Propaganda e Marketing ${ }^{1}$ (ESPM, 2018). Em dezembro de 2012, a Escola de Design Thinking ${ }^{2}$ (EDT) foi inaugurada, oferecendo um curso de Imersão em Design Thinking, com duração de quatro meses (PROJETO DRAFT, 2018).

Por ser uma abordagem relacionada à resolução de problemas, o design thinking encontra-se intimamente ligado à inovação e, a discussão de sua aplicação, difundiu-se nos círculos de design e gerenciamento. Houve uma mudança na abordagem do termo, da teoria para a prática, e de sua crescente utilização como um método (WRIGLEY; STRAKER, 2017).

Verifica-se que o design perpassou por diferentes formas de procedimentos, abordagens e métodos ao longo dos anos, processos tais que foram mapeados pela escada dinamarquesa do design, desenvolvida pelo Danish Design Centre, demonstradas na Figura 1 (KRETZSCHMAR, 2003 apud WRIGLEY; STRAKER, 2017).

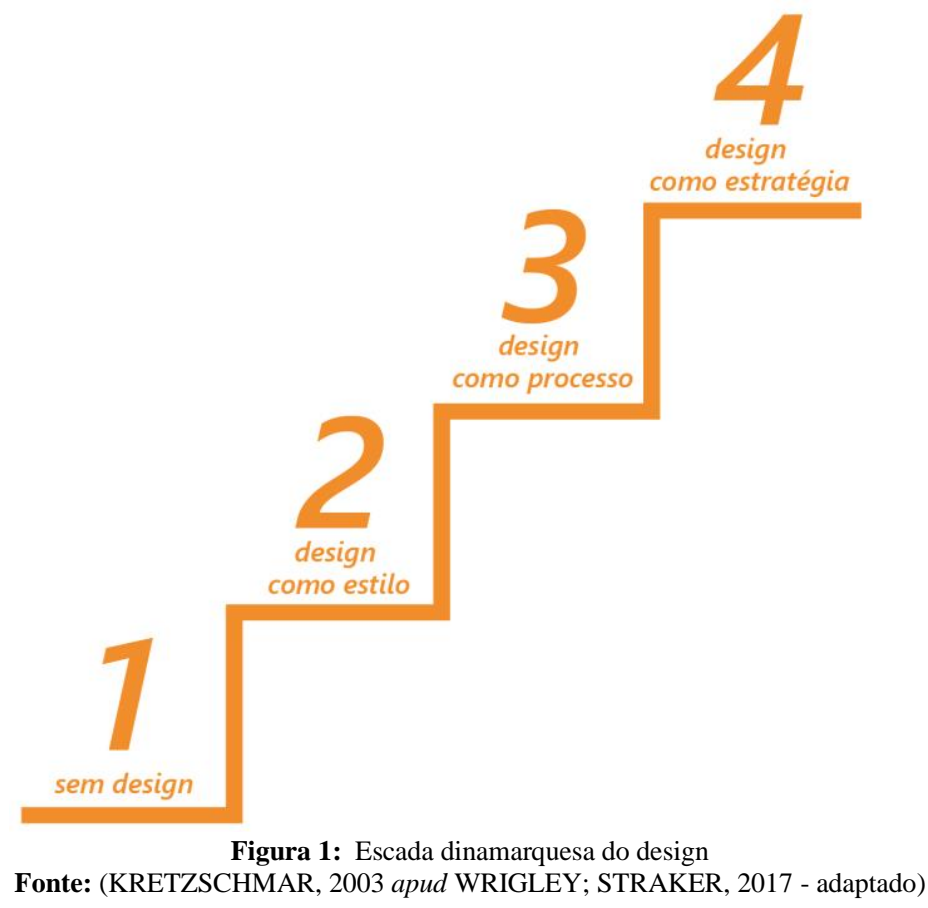

1 Localizada em São Paulo, Brasil

2 Com sua primeira sede localizada em São Paulo, Brasil 
Baseadas no modelo dinamarquês, Wrigley e Straker (2017) desenvolveram a Escada Educacional do Design, que ilustra os estágios pedagógicos no desenvolvimento do design thinking.

Dessa forma, o objetivo deste estudo é investigar quais os níveis de aplicação do design thinking nos estudos publicados nos Anais do Congresso Brasileiro de Pesquisa e Desenvolvimento em Design (P\&D), por ser o maior evento científico da área, com uma periodicidade histórica que contempla o recorte de tempo pesquisado. Os artigos foram avaliados por meio dos níveis descritos na Escada Educacional do Design (WRIGLEY; STRAKER, 2017), buscando verificar quais destes estão representados no evento. Partiuse da hipótese de que, por ser um tema relativamente novo no país, os níveis 1 e 2 da escada sejam os mais abordados.

Pretende-se, com este estudo, contribuir com a disponibilização de dados acerca do tema, como: abordagens e categorias das pesquisas publicadas, área do design inserida, universidades e regiões do Brasil, e os principais pesquisadores, ou seja, um panorama técnico, sem contudo, aprofundar discussões sobre sua funcionalidade, que pode ser discutida em futuras pesquisas.

Como resultado, obteve-se a visualização dos níveis trabalhados da Escada Educacional do Design, permitindo a identificação de lacunas para novas pesquisas, além de se obter um panorama da disciplina no congresso.

\section{Design Thinking}

O termo design thinking foi mencionado pela primeira vez em 1992, por Richard Buchanan, no artigo Wicked Problems in Design Thinking (PINHEIRO; ALT, 2017), contudo, esse conceito vem se desenvolvendo ao longo do último meio século por diversos estudiosos.

Buchanan cita quatro frentes de evolução do design que já não permitem que o mesmo fique restrito a uma disciplina, e sim ganhe o potencial de abordagem. São elas:

- o design ganha força na comunicação visual, estendendo seu foco da parte gráfica para a fotografia e a televisão.

- o design de produtos ganha também um olhar fundamental voltado para a função, indo buscar na engenharia e ciências sociais razões para as coisas existirem.

- o design aplicado a serviços, trazendo para o desenho de processos o seu pensamento holístico e foco nas pessoas.

- o design como abordagem na construção de melhores ambientes para as pessoas viverem e trabalharem. (PINHEIRO; ALT, 2017, p.48)

De definição complexa, o termo é amplamente descrito como uma abordagem, processo ou modelo mental (COSTA; SCALETSKY,2010; ECHOS, 2018; PINHEIRO; ALT, 2017; SERRAT, 2017), referindo-se à maneira do designer pensar, que utiliza o pensamento abdutivo (VIANNA et al., 2012) e a capacidade de combinar empatia, criatividade e racionalidade para analisar e adequar soluções a contextos particulares (WRIGLEY; STRAKER, 2017). 
Nesse processo, conforme o Design Council UK (2019), o designer passa por algumas etapas não lineares, mas iterativas: o de expandir o entendimento sobre o desafio enfrentado e o de refinar o conhecimento adquirido para encontrar novas associações e significados. O Design Council atribuiu a esse processo o nome de Duplo Diamante (Figura 2).

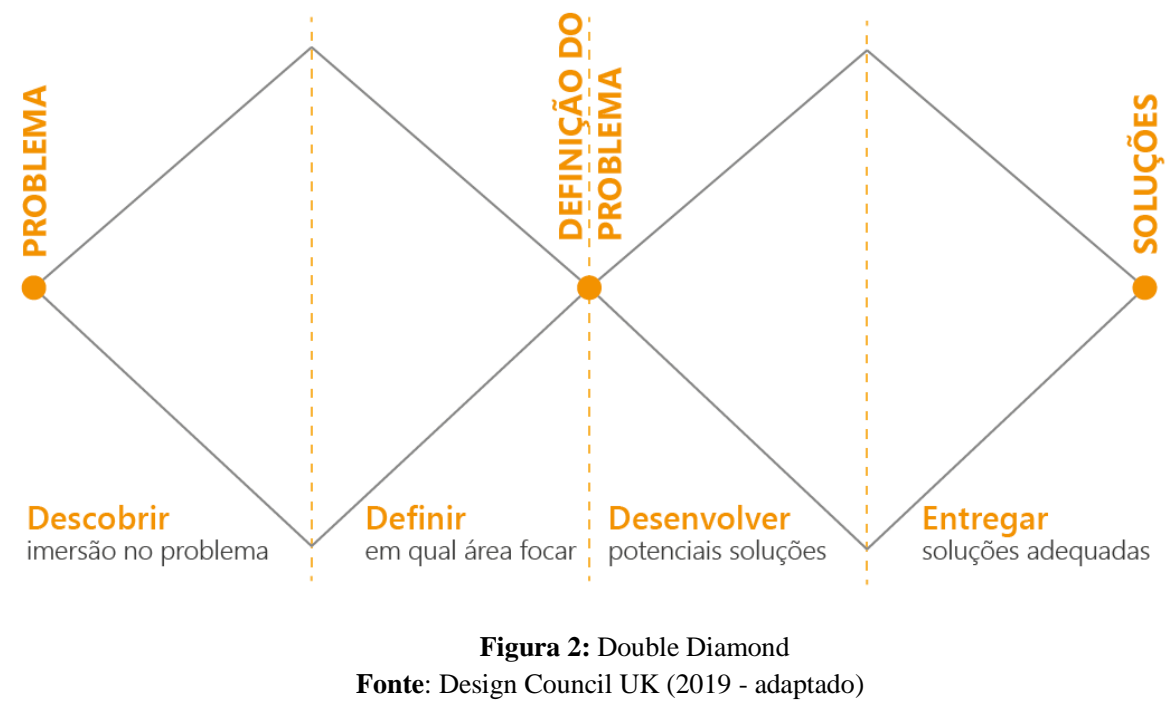

As linhas divergentes do modelo representam o momento de expandir o conhecimento, e as convergentes, o momento de fazer escolhas e refinar informações. Verifica-se metodologias similares em diversos escritórios de inovação por meio do design, tais como a D.School, Frog Design, LiveWork e IDEO (Figura 3). 


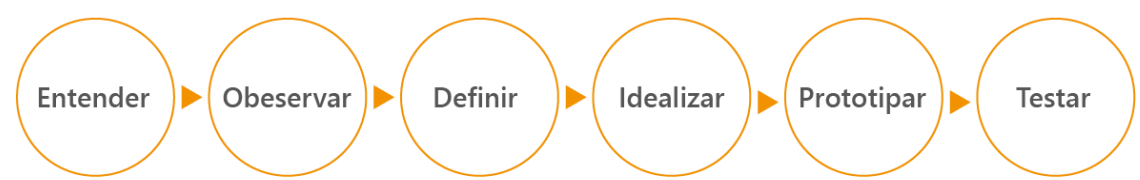

D.School: Compreender, Observar, Definir, Idealizar, Protipar, Testar

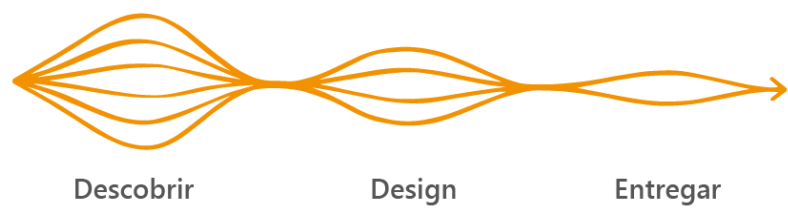

Frog Design: Descobrir, Projetar, Entregar

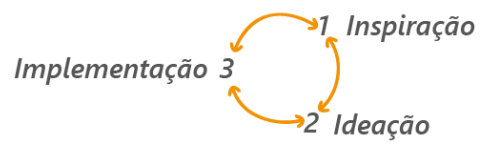

Ideo: Inspiração, Ideação, Implementação

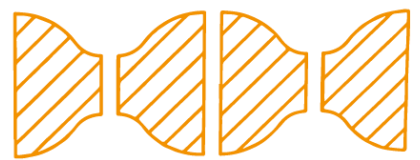

Insights Ideias Protótipos Realização

Live|Work: Insights, Ideias, Protótipos, Realização

Figura 3: Metodologia do Design Thinking em consultorias de design

Fonte: Pinheiro; Alt (2017, p.45 - adaptado)

Além desse processo em comum, a abordagem do design thinking preza pelo projeto centrado no ser humano, utilizando-se de ferramentas que permitam a imersão na realidade do usuário. Por isso, termos como empatia, cocriação, colaboração, experimentação, teste com usuários, são frequentes em projetos que o utilizam.

\section{A Escada Educacional do Design}

Inspirada na Escada Dinamarquesa do Design, descrita anteriormente, Wrigley e Straker (2017), desenvolveram a Escada Educacional do Design, que ilustra os estágios pedagógicos no desenvolvimento do design thinking. 
Após pesquisa com instituições de ensino de design da Dinamarca e outros países, sobre o conteúdo e a forma de ensino, emergiram cinco temas-chave: teorias, métodos e filosofias; foco no produto; gerenciamento de design; gerenciamento de negócios; e desenvolvimento profissional.

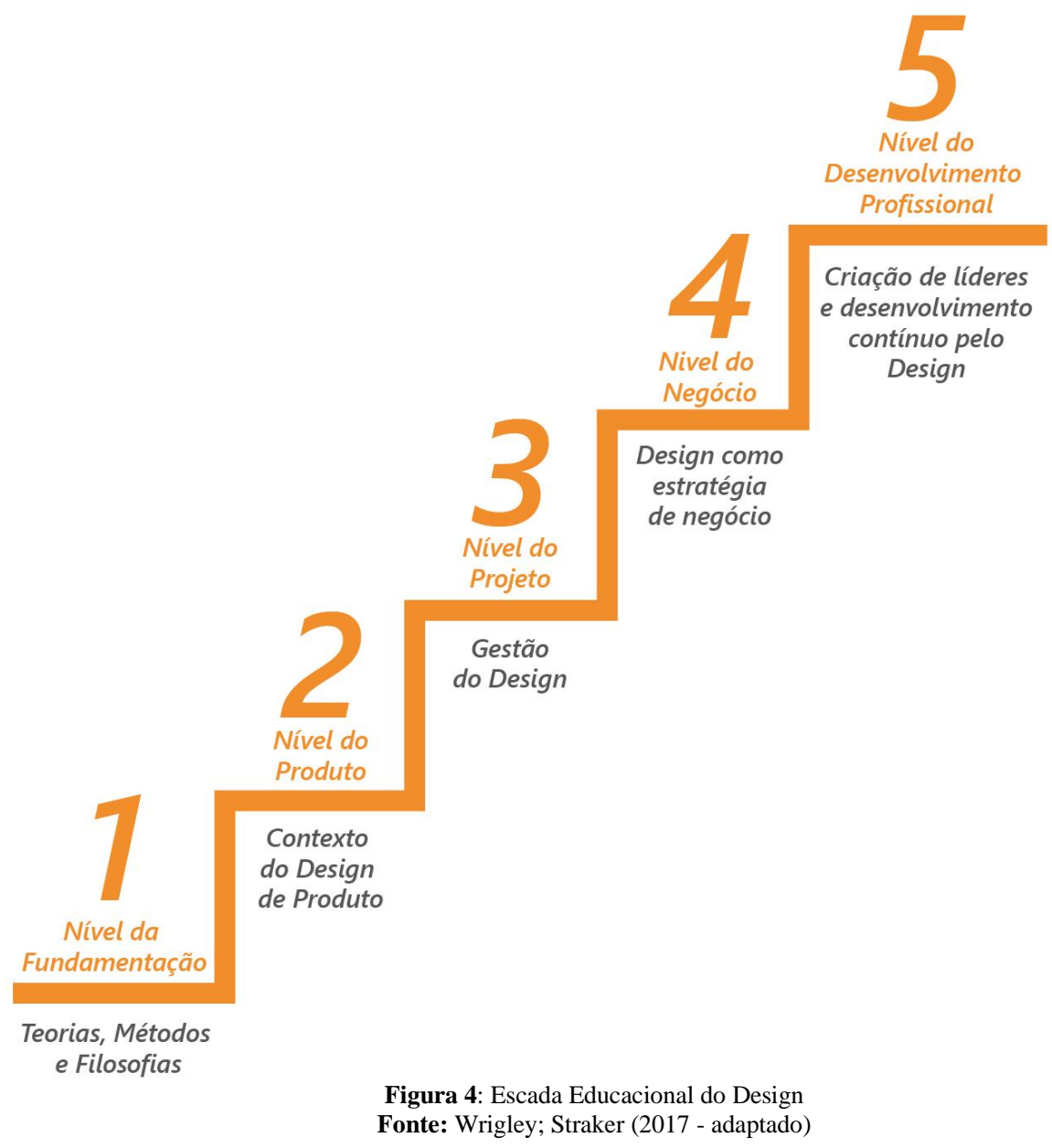

- Nível um (teorias, métodos e filosofias): fornece aos alunos o conhecimento da história, evolução e uso do design thinking. Ilustra o processo de design thinking, empregando e utilizando suas metodologias, filosofias e práticas reflexivas.

- Nível dois (foco no produto): coloca-se o design thinking no contexto do design do produto. Fornece a aplicação prática de métodos e processos de design thinking para resultados tangíveis no projeto e entrega de produtos e serviços.

- Nível três (gerenciamento de design): faz a ligação entre o design thinking e a gestão de design focados no produto. A abordagem é aplicada a fatores que influenciam um contexto de design mais amplo, como a situação do mercado e as decisões de marca.

- Nível quatro (gerenciamento de negócios): eleva o design thinking à área de estratégia de negócios e incorpora design, negócios e tecnologia no desenvolvimento de novos modelos de negócios e previsão estratégica.

- Nível cinco (desenvolvimento profissional): remove o design thinking de um contexto específico e visa desenvolver as habilidades pessoais e profissionais de um 
aluno/profissional. Utiliza a abordagem para demonstrar a importância de desenvolver habilidades para reconhecer oportunidades e nutrir o processo de levar ideias inovadoras à fruição.

De acordo com as autoras, os níveis da escada fornecem os vários tópicos, práticas de avaliação e modos de aprendizagem utilizados no ensino do design thinking, indicando uma progressão no conhecimento do tema.

O primeiro nível fornece a base para o design thinking como um método pelo qual os alunos adquirem habilidades em análise crítica e reflexão que lhes permitem criar seu próprio processo de design.

O segundo nível posiciona o design thinking como um processo para o desenvolvimento de novos produtos, usando uma abordagem centrada no ser humano para compreender as necessidades do usuário no nível do projeto.

O terceiro nível coloca o design thinking em um contexto mais amplo, considerando o gerenciamento de produtos e as especificações não relacionadas ao produto, incluindo marketing, questões financeiras, requisitos ambientais e o gerenciamento do lançamento de um produto.

O quarto nível foca no uso do design thinking em inovação e estratégia de negócios. Todos os elementos de um negócio, desde os planos de negócios até a previsão, são abordados nesta categoria.

O quinto nível contextualiza o indivíduo, dentro do conteúdo do curso, com o objetivo de criar líderes que reconheçam a importância do design thinking, e que possam nutrir e apoiar novas ideias.

Com base nessa pesquisa, o design thinking tornou-se objeto de estudo para compreender em qual nível educacional se encontram as pesquisas no maior congresso de design do país, o P\&D.

\section{Material e Métodos}

Para este estudo utilizou-se a técnica da Revisão Sistemática de Bibliografia, que tem como base a aplicação de métodos explícitos e sistemáticos, para filtrar o viés e auxiliar na identificação das evidências que se encaixam em critérios preestabelecidos para responderem uma questão de pesquisa (CONFORTO, AMARAL e SILVA, 2011; KHAN et al., 2003).

Moher et al., (2015) enfatizam que uma abordagem metodológica predefinida por meio de protocolos é essencial para conduzir uma Revisão Sistemática, pois detalha o raciocínio em uma lista de verificação. Portanto, para nortear a pesquisa, foi escolhido o método de Khan et al. (2003) em que apresentam cinco principais etapas: formular o problema, identificar os trabalhos relevantes, avaliar a qualidade dos estudos, resumir as evidências e interpretar os resultados (Tabela 1). Ressalta-se ainda, a elaboração de dois protocolos seguindo as orientações da segunda e terceira fase. 


\begin{tabular}{|l|l|}
\hline $1^{a}$ etapa & Definir a questão de pesquisa \\
\hline $2^{a}$ etapa & $\begin{array}{l}\text { Identificar os trabalhos relevantes; recursos utilizados, banco de dados, idiomas, critério de seleção } \\
\text { dos termos de busca, inclusão ou exclusão de termos devem ser registrados }\end{array}$ \\
\hline $3^{a}$ etapa & $\begin{array}{l}\text { Avaliação da Qualidade dos estudos; definir critérios para uma avaliação refinada que } \\
\text { posteriormente ajudará avaliar as intercorrências e descobrir lacunas para pesquisas futuras }\end{array}$ \\
\hline $4^{a}$ etapa & Resumir as evidências; síntese dos dados analisados por meio da tabulação dos critérios de avaliação \\
\hline $5^{a}$ etapa & Interpretar os resultados; análise e cruzamento dos resultados e pontos fortes e fracos das evidências \\
\hline
\end{tabular}

Tabela 1: Cinco Etapas para realizar uma revisão sistemática

Fonte: adaptado de Khan et al. (2003)

$\mathrm{Na}$ primeira etapa foi definido o seguinte problema de estudo: "em qual nível educacional se encontra o design thinking no congresso P\&D?" A fonte primária de busca dos trabalhos (segunda etapa), foi o Congresso Brasileiro de Pesquisa e Desenvolvimento em Design, por ser o maior evento científico de design do país, consequentemente, apresenta diversos assuntos na área.

Com isso, foi definido um critério de recorte da edição de 2002 a 2016. Anteriormente a 2002, os anais eram apenas impressos, o que dificultou o acesso aos mesmos, contudo, acredita-se que tal recorte não tenha prejudicado a pesquisa, pois apesar do design thinking surgir em 1992, no Brasil, ele ganhou maior relevância a partir de 2010, com o lançamento do primeiro livro sobre o tema. Não foram analisados os anais de 2018, pois à época de conclusão desta pesquisa, eles não estavam disponíveis.

Como forma de filtrar os trabalhos de acordo com o objeto de estudo, foi estabelecido um critério de busca por termos existentes em literatura do design thinking: "Design Thinking", "Design de Negócios" e "Design Estratégico".

O termo "Gestão do Design" não foi incluído por ser uma ferramenta com foco na organização para gerenciar todos os processos, incluindo identidade corporativa, prazos, ergonomia no ambiente de trabalho entre outros, que visam criar uma cultura organizacional. Já o design thinking usa métodos de design e a sensibilidade do designer para identificar problemas e solucioná-los fazendo uma imersão no problema junto ao público alvo (DEMARCHI, FORNASIER, MARTINS, 2011).

A gestão do design é uma atividade mais ampla, com viés administrativo e operacional. $\mathrm{O}$ design thinking possui uma visão mais humanista, apresentando uma abordagem experimental e criativa, permitindo que os usuários contribuam na geração de ideias. Com isso, agrega valor para o cliente tornando-se uma estratégia de negócio. Desta forma, o termo "Design de Negócios" foi incluído. Ele foi selecionado também por haver literatura sobre o design thinking com essa tradução para o português.

O "Design Estratégico" propõe identificar novas oportunidades de mercado, interpretá-las e traçar caminhos para orientar a ação organizacional e projetual, criando uma cadeia de valor para inovar o sistema-produto (DEMARCHI, FORNASIER, MARTINS, 2011; DE MORAES, 2010). Por ser uma disciplina relativamente nova no Brasil, e por haver semelhanças com a abordagem do design thinking, além permitir que se utilize dele no processo, foi decidido a manutenção do termo "Design Estratégico" na 
busca, pois chegou-se à conclusão de que eles ainda causam certa confusão em sua aplicação e não estão ainda suficientemente diferenciados.

Por fim, quando os termos "Design Thinking, "Design de Negócios" e "Design Estratégico" aparecerem no título, nas palavras-chave ou no resumo dos trabalhos, eles foram lidos na íntegra e catalogados no protocolo 1, denominado "Relação dos Artigos Analisados" (Tabela 02).

\begin{tabular}{|l|c|c|c|c|c|}
\hline \multicolumn{7}{|c|}{ Relação dos Artigos Analisados } \\
\hline Edição dos Anais & Página & Número do Trabalho & Título & Autores & Observações Gerais \\
\hline \multicolumn{6}{c|}{ Tabela 2: Relação dos artigos analisados } \\
Fonte: Os autores (2019)
\end{tabular}

Este primeiro protocolo teve como objetivo catalogar as informações básicas dos trabalhos analisados como: edição do evento e página do trabalho nos anais, número do trabalho - corresponde a um código de identificação definido pelos autores -, título, autores e observações gerais - trata-se de um resumo das principais informações do trabalho.

A terceira etapa corresponde à definição das categorias de qualidade, que auxiliaram na análise do conteúdo, para posteriormente sintetizá-las. Assim, foi criado o protocolo 2 (Figura 5), com critérios subdivididos em grupos como: número do trabalho definido na "relação dos artigos analisados" ou protocolo 1; termo de busca encontrado - "Design Thinking", "Design de Negócios", "Design Estratégico". O conteúdo: relacionado ao mercado ou ensino, a instituição de ensino: pública ou privada; o tipo de pesquisa referente a: dissertação, tese, artigo ou projeto de pesquisa; com abordagem teórica ou aplicada. A região do Brasil que foi desenvolvida, o nível de design thinking segundo a Escala Educacional (WRIGLEY; STRAKER, 2017) e por fim, a área da especialidade do design: Teoria do Design, Design Thinking, Design Estratégico, Gestão do Design, Educação/ Ensino, Design e Inovação e Branding.

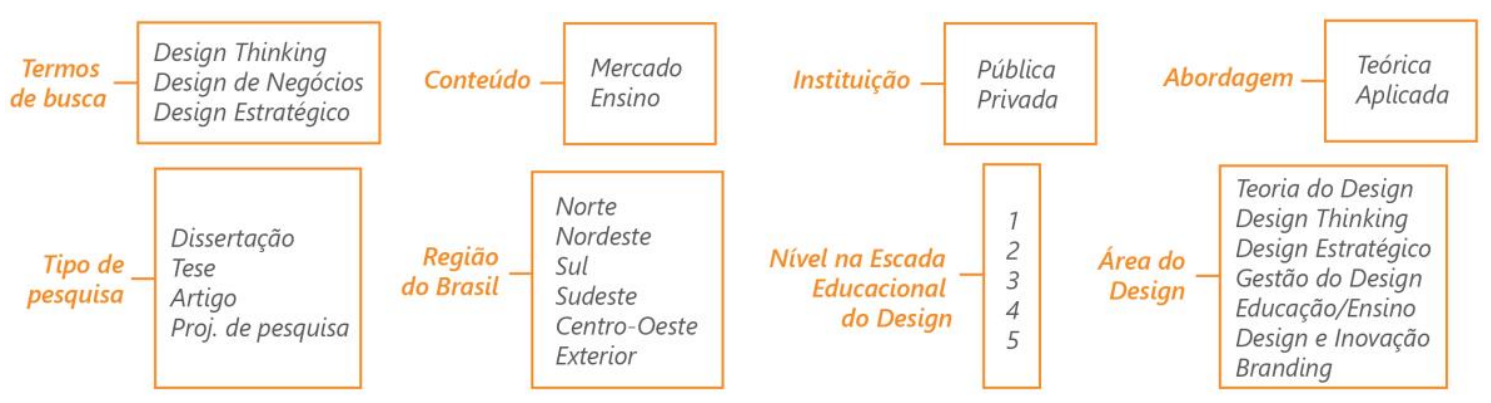

Figura 5: Protocolo 2 Fonte: Os autores (2019)

Após, foi realizada busca e análise dos trabalhos nos anais de 2002, 2004, 2006, 2008, 2010, 2012, 2014 e 2016. Foi realizada a tabulação das respostas por meio do cruzamento dos critérios apresentados no protocolo 2 e posteriormente, a interpretação dos dados em forma de gráficos, para verificar se a questão de pesquisa foi respondida. 


\section{Resultados e Discussões}

A busca realizada resultou em 113 artigos com os termos pesquisados nos títulos, palavras-chave ou resumo. Mas, 42 foram descartados, por tratarem de assuntos que não possuem relação com o método de design thinking. As edições de 2002 e 2006 possuem quatro trabalhos com os termos de busca, mas foram desconsiderados por conta do conteúdo. Restaram 71 artigos referentes às edições de 2004, 2008, 2010, 2012, 2014 e 2016, 70 pertencentes à categoria artigo completo e 1 pôster/resumo.

Dos 4108 trabalhos das edições analisadas, 1,72\% (71) estão relacionados ao tema pesquisado. O Gráfico 1 apresenta a evolução no número de publicações por edição.

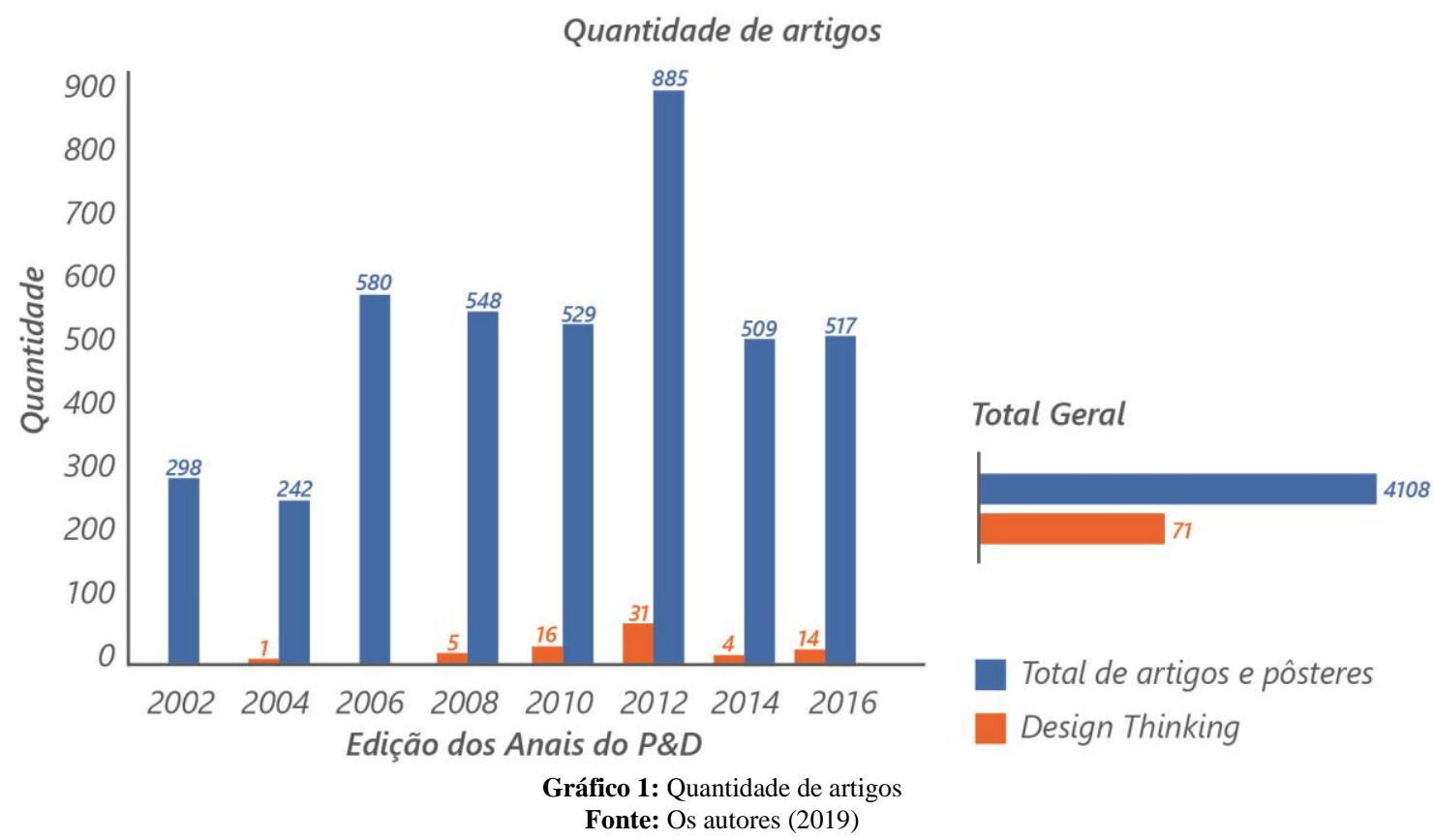

Em 2002 houve 298 publicações, mas nenhuma sobre design thinking. Em 2004, houve 242 artigos e apenas um sobre o tema. Em 2006, a quantidade de publicações dobrou (580) em relação à edição anterior, porém nenhuma se refere ao design thinking. Em 2008, 5 dos 548 (0,91\%), foram sobre o termo pesquisado. Esse fato coincide com a publicação na Harvard Business Review de um artigo escrito por Tim Brown abordando o tema, que se popularizou com a publicação do seu livro, Change by Design no ano seguinte (2009).

Em 2010, apesar da diminuição do número total de publicações (529), aumentaram os relacionados ao assunto, com 16 (3\%). A edição de 2012 apresenta o maior número de artigos publicados entre os anais analisados (885), e, destes, 31 estão relacionados ao design thinking. Esse aumento pode estar relacionado com o início das publicações em português sobre o tema, que ocorreram a partir de 2010. Constata-se que 2012 foi o ano com maior número de artigos, e consequentemente, sobre o termo pesquisado. Também, proporcionalmente, em 2012 houve a maior concentração de trabalhos acerca do tema, com $3,5 \%$ do total.

Com a diminuição das publicações em 2014 (509), também houve declínio nos relacionados ao tema - apenas 4 . Tal fato mostrou-se intrigante, uma vez que o congresso 
desse ano foi realizado em Gramado (RS), região onde mais houve artigos publicados sobre o tema, e esperava-se que esse número fosse mais expressivo.

Em 2016 houve 517 artigos, com 14 sobre o tema, representando 2,7\% do total.

\section{Termos de busca}

O termo "Design Estratégico" apareceu nas edições de 2004, 2008, 2010 e 2012. Já o "Design Thinking" começou a ganhar representatividade em 2010 e, em 2016, percebe-se um crescente significativo (Gráfico 2). Esses dados podem estar relacionados ao primeiro livro publicado no Brasil sobre o assunto, no ano de 2010. Em decorrência, à primeira escola de Design Thinking do país, em 2012.

"Design de Negócios" aparece em um dos 71 artigos, na edição de 2016. Ressalta-se que, em alguns trabalhos, houve a ocorrência de mais de um termo, portanto, a somatória do total dos termos é maior que a somatória do número de artigos analisados.

Do total de termos encontrados, 65,7\% referem-se ao "Design Estratégico", 32,8\% ao "Design Thinking" e apenas 1,36\% ao "Design de Negócios". Com esse resultado, pode-se inferir que o design thinking, apesar de poder ser utilizado pelo design estratégico, ainda é bastante confundido com o mesmo, visto que aparece mais que o próprio termo que fundamenta esta pesquisa. Entretanto, verifica-se que eles caminham para uma diferenciação mais acentuada, e nos próximos anos, os resultados indicam a tendência de se fixarem como dois termos distintos com abordagens e aplicações singulares.

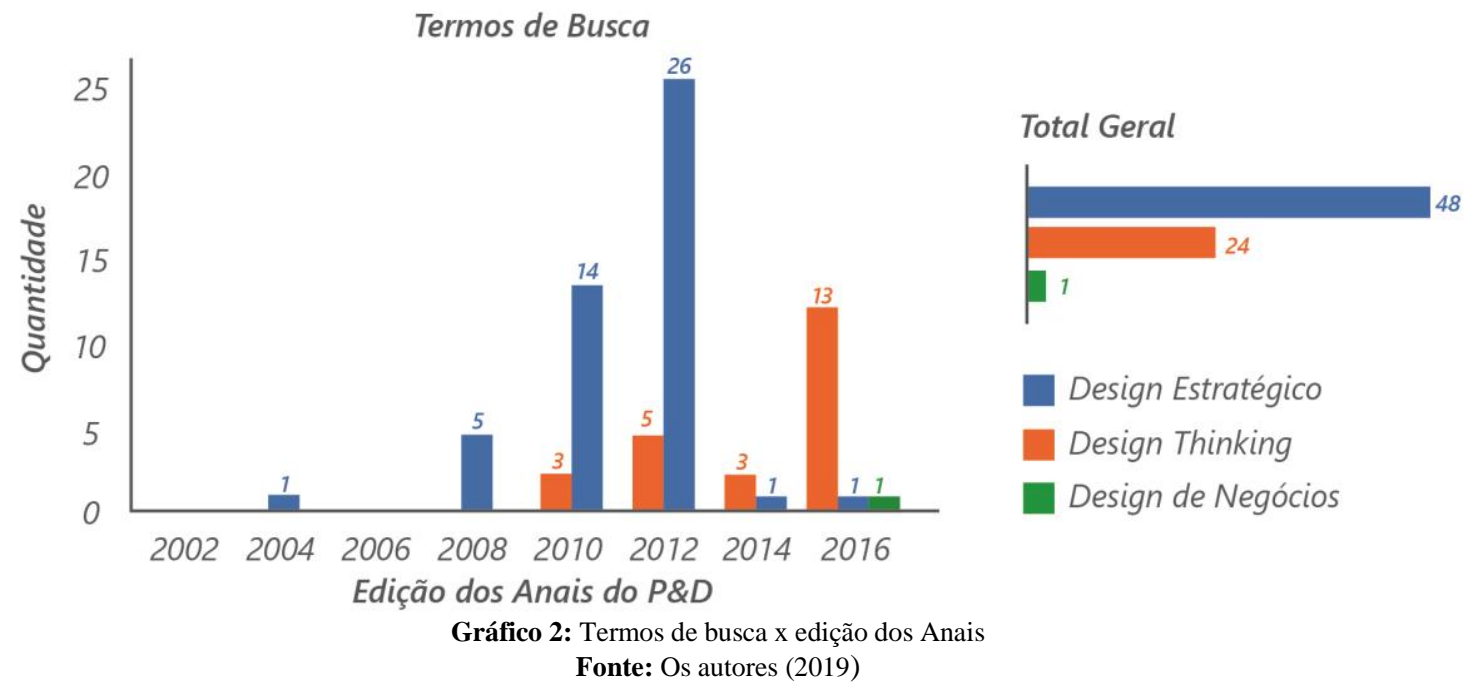

\section{Tipos de pesquisa}

Em relação aos tipos de pesquisa (Gráfico 3), 85,9\% referem-se a artigos científicos, $9,85 \%$ a projetos de pesquisa, $2,8 \%$ a resultados de dissertações e $1,4 \%$ de teses. Como a grande maioria dos estudos refere-se a artigos, é possível identificar lacunas de pesquisa para o termo no Banco de Teses e Dissertações da Capes e Periódicos, o que enriqueceria o resultado deste estudo, complementando-o.

Outra lacuna seria a pesquisa do termo em congressos de outras áreas que se utilizam da abordagem do design thinking, como a administração e o marketing. 


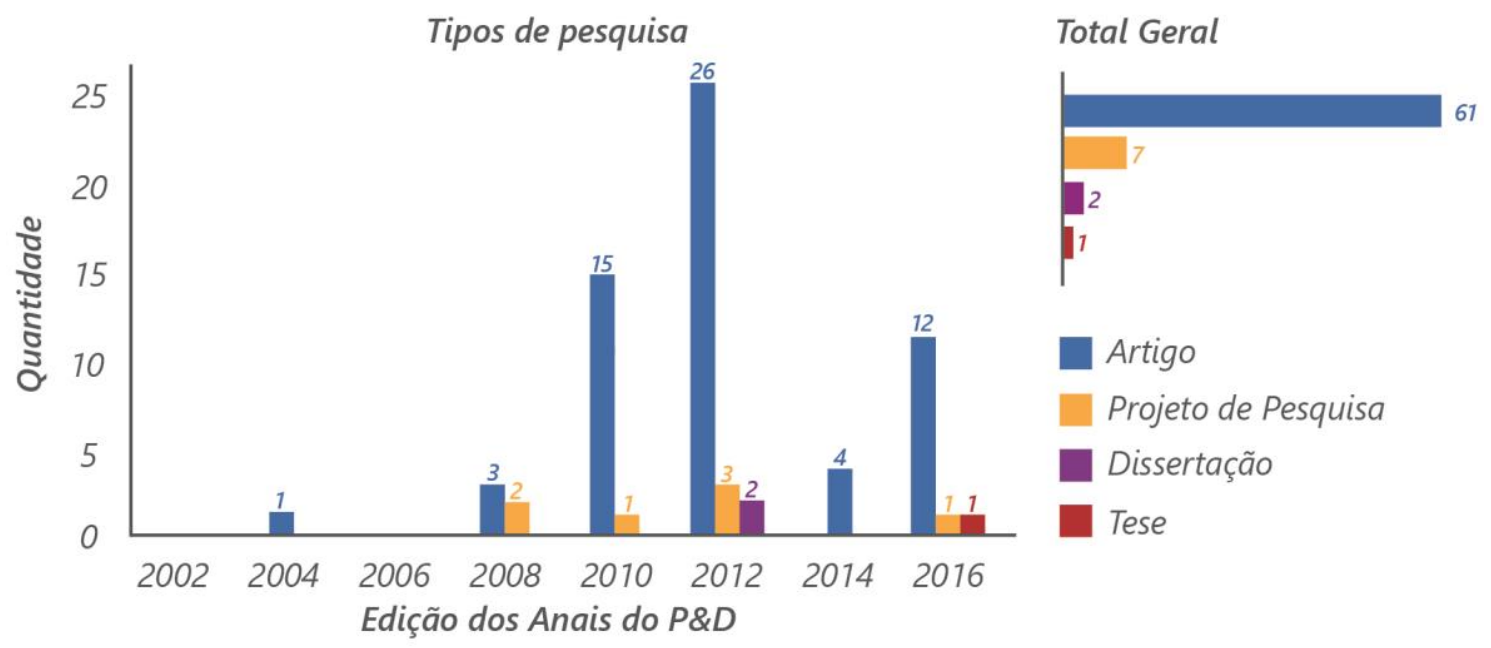

\section{Gráfico 3: Tipos de pesquisa} Fonte: Os autores (2019)

\section{Tipos de abordagens}

O Gráfico 4 apresenta o resultado das análises segundo o viés de abordagem dos trabalhos, caracterizando-os como Mercado, voltado para a indústria ou ensino, com foco em atividades do ambiente acadêmico. Outra variável presente no Gráfico 4 são os trabalhos com aspecto teórico ou aplicado.

As edições de 2004 a 2012 apontam um aumento nos números de trabalhos com abordagem de mercado. Entretanto, no ano de 2014 houve uma queda significativa e depois uma nova alta. $\mathrm{O}$ mesmo ocorreu com artigos relacionados aos assuntos de ensino.

Os trabalhos de caráter teórico, aparecem em todos os anais pesquisados, com algumas variações na edição de 2014. Já os que possuem conteúdo aplicado, surgem na edição de 2008 e também apresentam uma oscilação em 2014.

Percebe-se que os temas encontram-se bem divididos e representados no P\&D. Alguns dos artigos continham mais de uma categoria de avaliação, por isso, a soma total é maior que a soma do número de artigos.

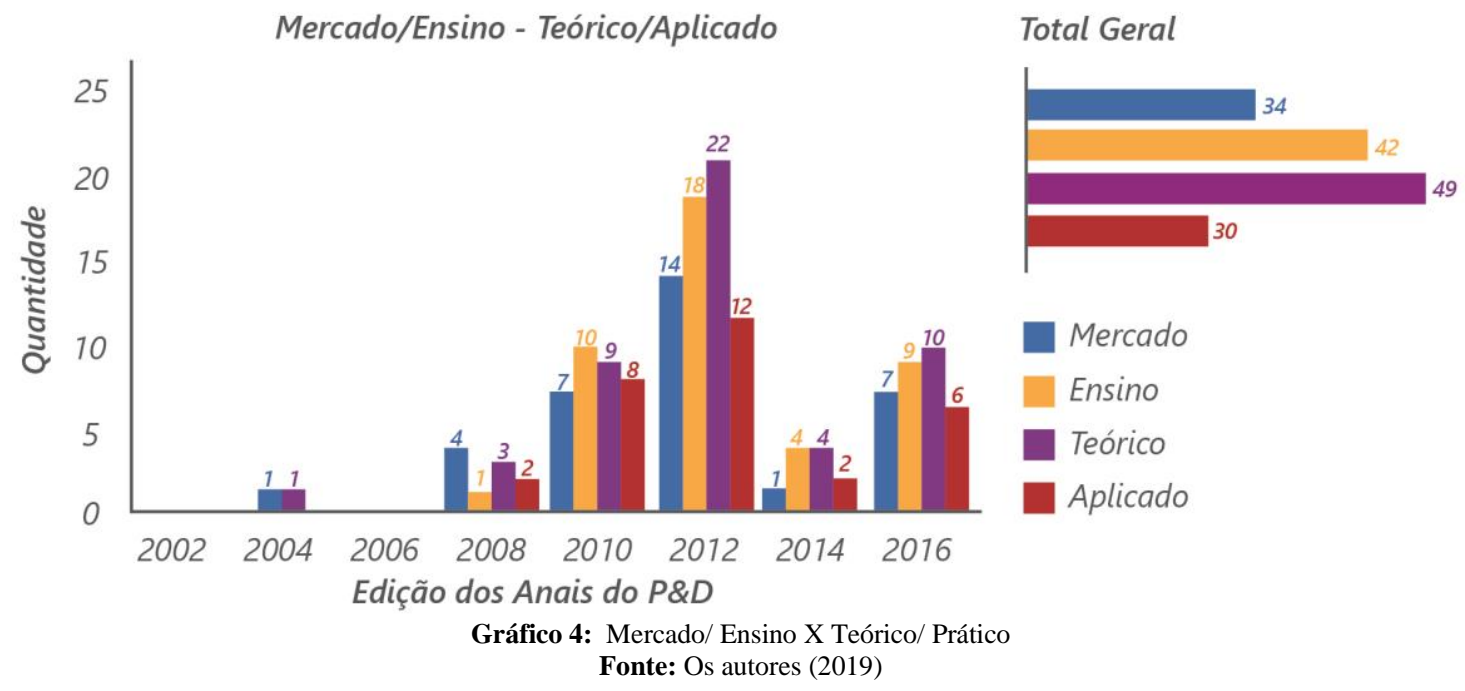




\section{Regionalidade}

Os trabalhos filiados às instituições de ensino pública e privada foram contabilizados em relação às regiões do Brasil e representados no Gráfico 5. Destaca-se a região Sul com 55 publicações, 17 trabalhos de instituições públicas, com destaque para a Universidade Federal de Santa Catarina (UFSC) com 7 artigos, e 38 trabalhos de instituições privadas, com ênfase para a Unisinos, com 33 publicações.

O Sudeste aparece em segundo lugar com 23 publicações, 12 trabalhos de instituições públicas e o restante de instituições privadas. Dentre as instituições públicas, a Universidade Estadual de Minas Gerais (UEMG) e a Universidade de São Paulo (USP) apresentam o maior número de artigos. Já entre as instituições privadas, PUC-Rio possui 5 artigos e a empresa MJV - Inovação e Tecnologia, 4 artigos.

O Nordeste possui 4 publicações, todas da Universidade Federal de Pernambuco (UFPE).

O Norte tem 2 publicações, ambas da Universidade privada Fundação Centro de Análise, Pesquisa e Inovação Tecnológica (FUCAPI).

Dos 71 trabalhos analisados, 3 são de instituições de ensino privadas fora do país, mas em parceria com universidades brasileiras. Do mesmo modo, ocorreram parcerias entre as instituições brasileiras, por isso a soma dos números de artigos representados no Gráfico 5 é maior do que o total de artigos analisados. 


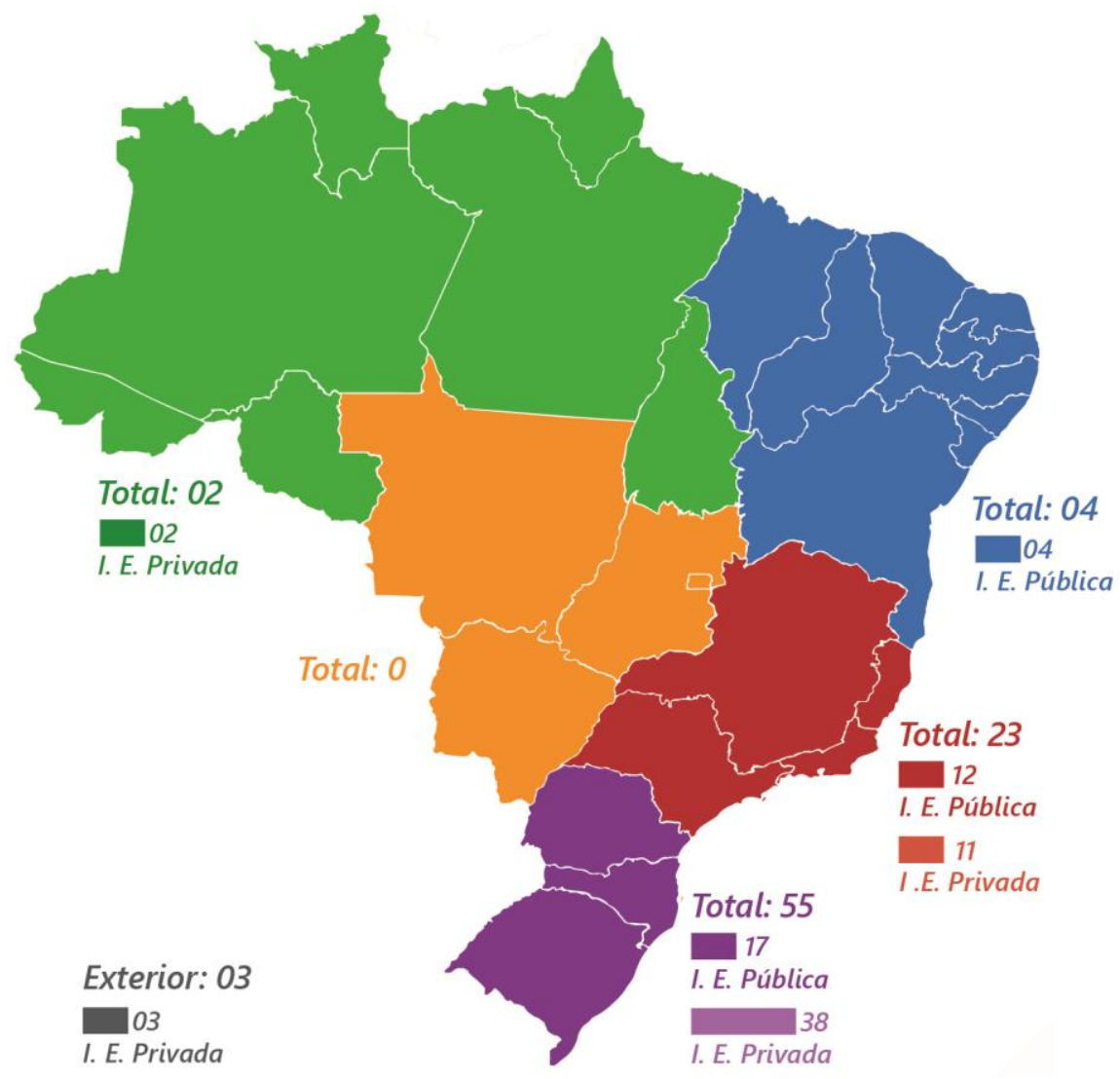

SUL

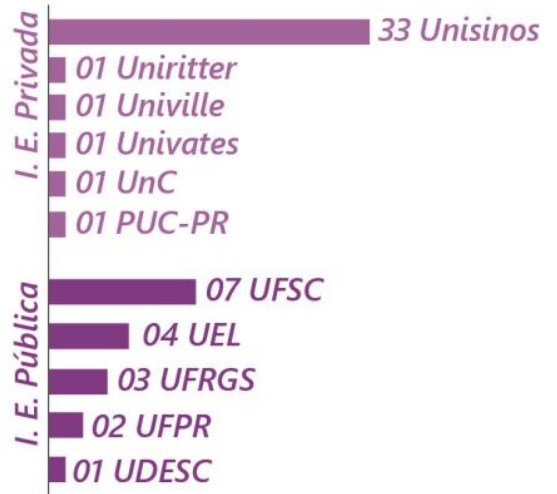

NORDESTE

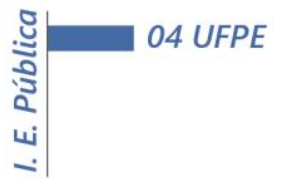

SUDESTE

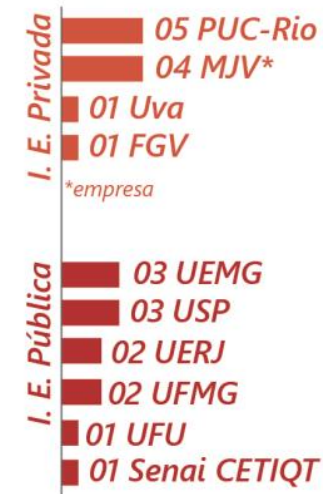

NORTE

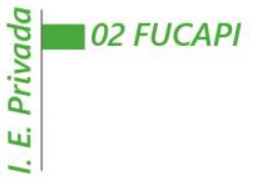

Gráfico 5: Publicações por instituições e regiões do Brasil Fonte: Os autores (2019)

\section{Autores}

Os autores que possuem maior número de publicações são REYES, Paulo e FRANZATO, Carlo, ambos com 8 artigos; também, SCALETSKY, Celso Carnos, aparece com 7 estudos. Os três autores são membros da instituição Unisinos, que se destaca em publicações sobre o tema. 


\section{Áreas do Design}

No que se refere às áreas do design, destaca-se o design estratégico, com 37 artigos. Em seguida o design thinking com 13, teoria do design com 8, educação e ensino com 6 , gestão do design e design e inovação com 5 cada e, branding com 4 (Gráfico 6).

A classificação dos artigos foi realizada pelos autores, agrupando temas semelhantes e de maior frequência para que não houvesse uma diversidade muito grande no número das temáticas. Portanto, tal classificação pode não corresponder aos eixos temáticos dos anais do congresso.

Na edição de 2010 para 2012 houve um aumento de publicações na área de design estratégico. Em contrapartida, a partir de 2012 começaram a aumentar trabalhos sobre design thinking e a diminuir os sobre design estratégico. Tal resultado pode ser efeito do melhor entendimento e diferenciação dos termos, além da sua maior divulgação no país.

Percebe-se uma grande variedade de temas que abordam o design thinking, pois ele pode ser utilizado em conjunto com vários outros métodos e ser aplicado a diversas disciplinas por se tratar de uma abordagem flexível.

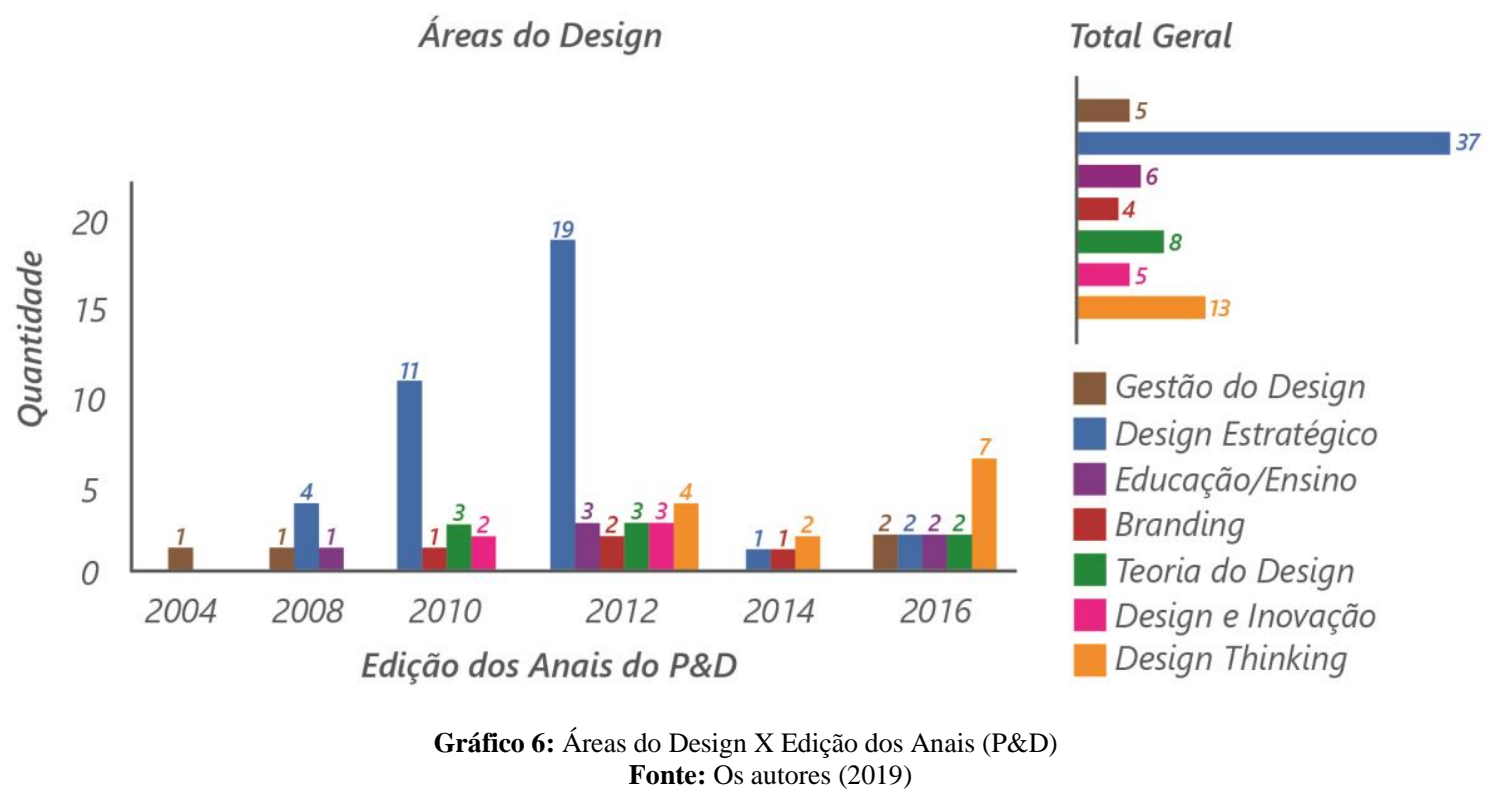

\section{Nivel Educacional do Design Thinking}

A maioria dos artigos publicados encontram-se no nível 1, principalmente, e também no nível 2 da Escada Educacional do Design. Há poucos nos níveis 3 e 4 e apenas um no nível 5.

As demais regiões do país têm poucas publicações sobre o tema, a maioria sendo em edições mais recentes do congresso, como pode-se ver no Gráfico 7.

Esse resultado permite inferir que o design thinking encontra-se mais disseminado nas regiões sul e sudeste do país, sendo recentemente abordado por instituições de outras regiões, o que também pode ser causa dos diversos níveis da escada educacional encontrados, uma vez que os níveis mais básicos (1 e 2) são os únicos que aparecem nas outras regiões do país. 


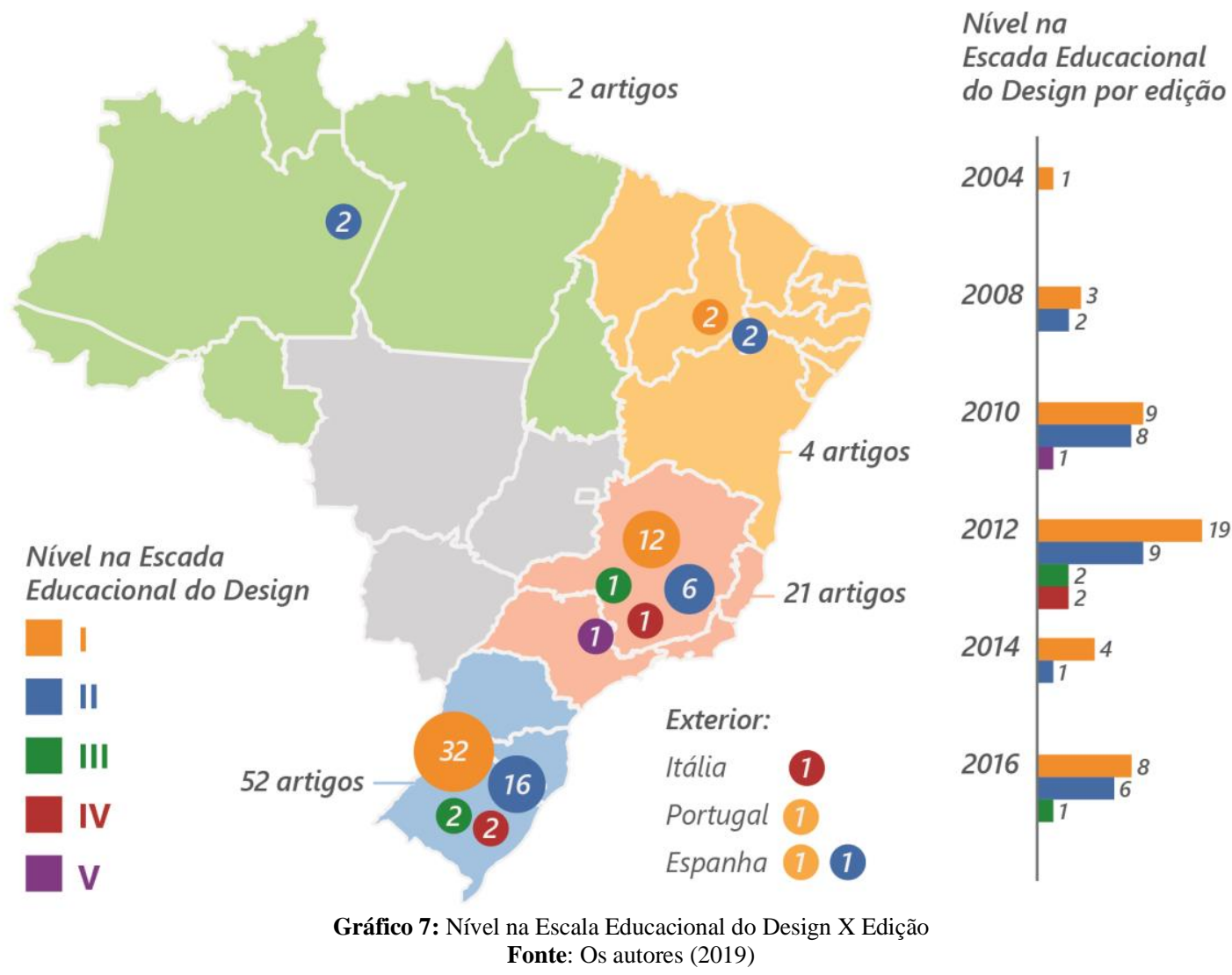

\section{Considerações finais}

Este trabalho apresentou uma Revisão Sistemática Bibliográfica nos anais de 2002 a 2016 do P\&D, analisando 71 artigos com conteúdo relacionado ao design thinking e comparou os resultados com a Escada Educacional do Design Thinking, identificando que os estudos publicados no país encontram-se no primeiro e segundo nível, relacionados com a teoria, história, filosofia e reflexão (1) e com o foco no produto (2). Apesar de haver 6 trabalhos relacionados aos outros níveis, há carência em pesquisas sobre os níveis de gerenciamento em design, negócios e desenvolvimento profissional.

Constatou-se que do total de artigos publicados em todos os anais analisados (4108), o design thinking representa apenas $1,72 \%$ desse total. Esse fato indica a necessidade de mais pesquisas sobre o tema, tanto numericamente, quanto em relação aos níveis da Escada Educacional do Design. Houve uma crescente do termo nos congressos de 2008 a 2012, com um declínio em 2014, fato que se mostrou intrigante para os pesquisadores. Entretanto, houve um novo aumento das publicações em 2016, e espera-se que, com a consolidação e melhor entendimento do termo, ele venha a ter mais representatividade, com novas pesquisas e abordagens em diferentes níveis (da escada educacional do design) e regiões do país.

A maioria dos artigos possui uma abordagem teórica, reforçando a hipótese de que o país encontra-se nos primeiros níveis da escada, em um momento de aprofundamento das teorias, métodos e instrumentos de aplicação do design thinking. 
Em relação às regiões do Brasil, o Sul se destaca, possuindo uma grande abrangência dos níveis da escada (1 ao 4), contudo, ainda não possui nenhuma publicação no nível 5.

O Sudeste vem logo em seguida, contudo, verifica-se uma produção irregular, bastante dispersa entre as universidades da região, mas que aborda todos os níveis da escada educacional.

O Nordeste, apesar de ter publicações em 2010, 2012 e 2016, possui apenas 4 artigos nos anais analisados, todos nos níveis 1 e 2 , pertencentes à mesma instituição, sugerindo uma suposta superficialidade do tema.

O Norte conta com apenas dois artigos publicados nos anais 2016, o mais recente analisado, indicando que o interesse sobre o tema é novo na região. Esses artigos são todos do segundo nível da escada.

O Centro-Oeste não tem nenhum artigo publicado.

Por fim, a Escala Educacional do design thinking mostrou ser uma ferramenta eficaz para mensurar o nível educacional da disciplina no Brasil e informar as abordagens mais complexas e desconhecidas da disciplina. Apesar de possuir uma irregularidade nas publicações, elas tiveram um aumento em 2016, o que indica que há interesse dos pesquisadores sobre o assunto, que, como relatado neste estudo, ainda pode ser muito explorado.

\section{Referências}

CONFORTO, E. C.; AMARAL, D. C.; SILVA, S. L. DA. Roteiro para revisão bibliográfica sistemática: aplicação no desenvolvimento de produtos e gerenciamento de projetos. In: Congresso Brasileiro de Gestão de Desenvolvimento de Produto - CNGDP, 8, n.1988, 2011. pp. 1-12. Anais... Disponível em: 〈http://vision.ime.usp.br/ acmt/conforto.pdf〉. Acesso em: 8 out. 2018.

CONGRESSO BRASILEIRO DE PESQUISA E DESENVOLVIMENTO EM DESIGN, 5, 2002, Brasília. CD- ROM: Anais $5^{\circ}$ P\&D Design. Brasília, 2002.

CONGRESSO BRASILEIRO DE PESQUISA E DESENVOLVIMENTO EM DESIGN, 6, 2004, São Paulo. CD-ROM: Anais $6^{\circ}$ P\&D Design. São Paulo, 2004.

CONGRESSO BRASILEIRO DE PESQUISA E DESENVOLVIMENTO EM DESIGN, 7, 2006, Curitiba. CD- ROM: Anais $7^{\circ}$ P\&D Design. Curitiba, 2006.

CONGRESSO BRASILEIRO DE PESQUISA E DESENVOLVIMENTO EM DESIGN, 8, 2008, São Paulo. CD- ROM: Anais $8^{\circ}$ P\&D Design. São Paulo, 2008.

CONGRESSO BRASILEIRO DE PESQUISA E DESENVOLVIMENTO EM DESIGN, 9, 2010, São Paulo. Anais eletrônico 9 $\mathbf{9}^{\mathbf{0}}$ P\&D Design. 2010. Disponível em: https://pt.scribd.com/document/337320667/Anais-9-Ped-2010 Acesso em: 15 dez. 2018.

CONGRESSO BRASILEIRO DE PESQUISA E DESENVOLVIMENTO EM DESIGN, 10 , 2012, São Luís. Anais eletrônico $\mathbf{1 0}^{\circ}$ P\&D Design. 2012. Disponível em: http://www.peddesign2012.ufma.br/anais/ Acesso em: 18 dez. 2018.

CONGRESSO BRASILEIRO DE PESQUISA E DESENVOLVIMENTO EM DESIGN, 11, 2014, Gramado. Anais eletrônico 11 ${ }^{\circ}$ P\&D Design. Blucher, 2014. Disponível em: http://www.proceedings.blucher.com.br/article-list/11ped-233/list\#articles Acesso em: 20 dez. 2018.

COSTA, F. C. X.; SCALETSKY, C. C. Design Management \& Design Estratégico: uma Confusão Conceitual? In: Congresso Brasileiro de Pesquisa e Desenvolvimento em Design, 9, 
2010, São Paulo. Anais... São Paulo: Blücher e Universidade Anhembi Morumbi, 2010. Disponível em: <https://pt.scribd.com/document/337320667/Anais-9-Ped-2010>. Acesso em: 15 dez. 2018.

DE MORAES, Dijon. Metaprojeto: o design do design. São Paulo: Blucher, 2010.

DEMARCHI, Ana Paula Perfetto; FORNASIER, Cleuza Bittencourt Ribas; MARTINS, Rosane Fonseca de Freitas. A Gestão de Design humanizada pelo Design thinking a partir de relações conceituais. Projética, v. 2, n. 1, p. 19-36, 16 dez. 2011.

DESIGN COUNCIL UK. The Design Process: What is the Double Diamond? Disponível em: <https://www.designcouncil.org.uk/news-opinion/design-process-what-double-diamond>. Acesso em: 6 jan. 2019.

ECHOS. Etapas do Design Thinking na prática | Echos. Disponível em: <https://escoladesignthinking.echos.cc/materiais/etapas-do-design-thinking-na-pratica/>. Acesso em: 5 jan. 2019.

ESPM. Design Thinking @ ESPM. Disponível em: <https://designthinkingespm.com.br/>. Acesso em: 7 out. 2018.

KHAN, K. S. et al. Five steps to conducting a systematic review. In: Journal of the Royal Society of Medicine, v.96, mar. 2003, pp. 118-121. DOI:10.1258/jrsm.96.3.118

KRETZSCHMAR, A. The Economic Effects of Design. In: Danish National Agency for Enterprise and Housing, Dinamarca, 2003. Disponível em: <https://boligejer.dk/file/1924/the_economic_effects_of_design.pdf $>$. Acesso em: 5 jan. 2019.

MOHER, D. et al. Preferred reporting items for systematic review and meta-analysis protocols (PRISMA-P) 2015 statement. Systematic Reviews, v. 4, n. 1, p. 1, 1 jan. 2015.

PINHEIRO, T.; ALT, L. Design Thinking Brasil: empatia, colaboração e experimentação para pessoas, negócios e sociedade. Rio de Janeiro: Alta Books, 2017.

PROJETO DRAFT. Inovação para um mundo melhor: este é o modelo de sucesso da primeira escola de Design Thinking do Brasil. Disponível em: $<$ https://projetodraft.com/inovacao-para-um-mundo-melhor-este-e-o-modelo-de-sucesso-daprimeira-escola-de-design-thinking-do-brasil/>. Acesso em: 7 out. 2018.

SERRAT, O. Design Thinking. In: SERRAT, O. (Ed.). Knowledge Solutions: Tools, Methods, and Approaches to Drive Organizational Performance. Singapore: Springer Singapore, 2017. p. 129-134.

VIANNA, M. et al. Design Thinking: inovação em negócios. 1. ed. Rio de Janeiro: MJV Press, 2012.

WRIGLEY, C.; STRAKER, K. Design Thinking pedagogy: the Educational Design Ladder. Innovations in Education and Teaching International, v. 54, n. 4, p. 374-385, 4 jul. 2017. DOI: 10.1080/14703297.2015.1108214.

\section{Sobre os autores}

Marina Granja Arakaki - marina.arakaki@unesp.br

Possui graduação em Design Gráfico pela Universidade Estadual de Londrina (2004) e mestrado em Estudos de Linguagens pela Universidade Federal de Mato Grosso do Sul (2014).

Doutoranda no Programa de Pós-Graduação em Design da Universidade Estadual Paulista Júlio de Mesquita Filho (2018). Tem experiência na área de Artes, com ênfase em Design, atuando principalmente nos seguintes temas: design gráfico, design editorial e identidades visuais.

Ana Cláudia de Abreu - a.abreu@unesp.br 
Possui graduação em Moda pelo Centro Universitário de Maringá (2013). Especialização em Moda, produto e educação pela Universidade Estadual de Londrina (2016) e Docência no Ensino Superior pelo Centro Universitário de Maringá (2016). Mestranda em Design pela Universidade Estadual Paulista.

Mariano Lopes de Andrade Neto - mlaneto@ gmail.com

Graduação em Desenho Industrial - Programação Visual pela Universidade Estadual de Londrina (2003), mestrado em Design pela Universidade Estadual Paulista Júlio de Mesquita Filho (2011), doutorado em Design pela Universidade Estadual Paulista Júlio de Mesquita Filho (2016). Graduando em Gestão da Tecnologia da Informação pela Universidade Estácio de Sá (2019). Atualmente é docente na UniFSP - Centro Universitário Sudoeste Paulista (2017). 